\title{
Defining the machining process for complex parts made from aluminium alloy extruded profiles by correcting raw material deviations
}

\author{
Ștefan Adrian Moldovan*, Mihai Banica, and Vasile Năsui \\ Technical University of Cluj-Napoca - North University Center of Baia Mare, Victor Babes, 62A, \\ Baia Mare, 430083, România
}

\begin{abstract}
In this paper we present technical solutions to define the machining process to create good products specific for aerospace industry made of aluminium alloy extruded profile. Those parts have very tight tolerances and it is necessary to create a robust system to ensure repeatability of machining processes.

The major factor which affects our machining process is internal stress effect of the aluminium alloy extrusions. To determinate this influence were made several tests, using parts for aerospace industry, those parts were measured through coordinate measuring machine and all data was recorded for statistical analysis. Based on input data was determinate a technical solution to eliminate deviations caused by raw material internal stress for obtaining parts in tolerance.

Through several tests and recording all dimensions changes during the milling process, was obtained good products and was ensured repeatability of the machining process.
\end{abstract}

\section{Introduction}

The major concern for our production is to maintain the production repeatability. In aerospace industry it's not space to make mistakes for this reason we need to create a robust system to can ensure our products quality. Starting a new project on S.C. Universal Alloy Corporation Europe S.R.L. factory, were determined a machining strategy to can machine parts made from aluminium alloy extrusions. Through several tests which was measured on the coordinate measuring machine and all data was recorded for statistical analysis. Creating the Ishikava diagram was determined all factors which affect the machining process in this factory (Figure 1). The aim for this research is to track and control the extrusion internal stress using input data's to determinate the proper machining strategy [1].

\footnotetext{
${ }^{*}$ Corresponding author: moldovan.stefanadrian@gmail.com
} 


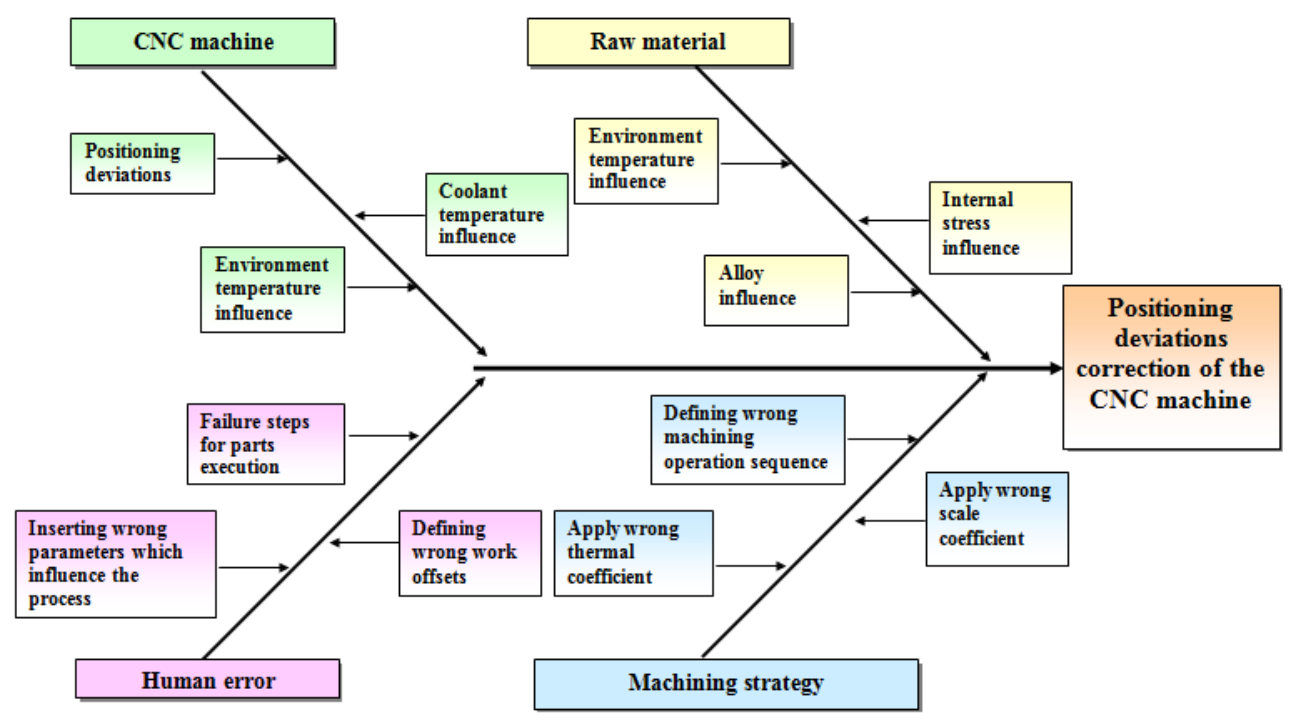

Fig. 1. Ishikawa diagram.

\section{Manufacturing methodology}

The machining process is performed in a normal processing unit, with climate control and the CNC (computer numerical control) machine used is Handtmann PBZ HD 600 (Figure 2). The CNC machine provides temperature compensation based on environment temperature fluctuation and also it is equipped with chiller system to maintain constant the coolant temperature [2].

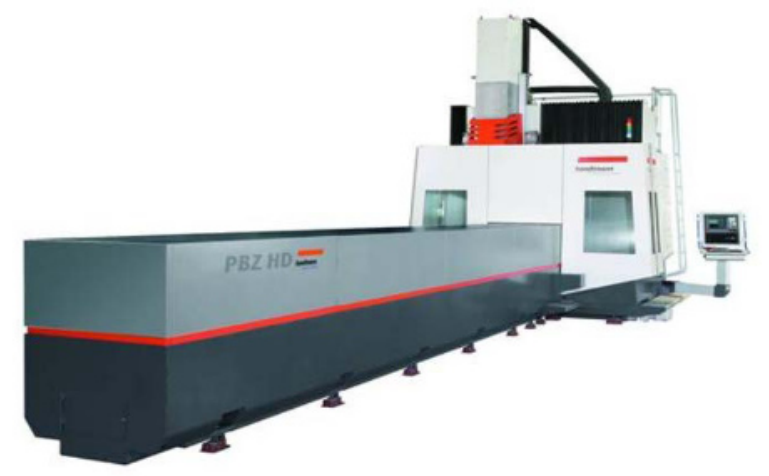

Fig. 2. Handtmann PBZ HD 600.

Developing optimum machining results with best accuracy, high material removal rates with $63 \mathrm{~kW}$ spindle power a maximum $30000 \mathrm{rpm}$. As well as short machining times and reduced cost per part are the outcomes. The HD machine provides full 5-axis simultaneous machining [3]. All machined parts specific for aerospace industry are validated by CMM (coordinate measuring machine), placed in a room without temperature control environment.

$\mathrm{CMM}$ is a device for measuring the physical geometrical characteristics of an object. The typical 3 "bridge" CMM is composed of three axes, X, Y and Z [4]. These axes are orthogonal to each other in a typical three-dimensional coordinate system. Each axis has a 
scale system that indicates the location of that axis. The machine will read the input from the touch probe, as directed by the operator or programmer. The machine then uses the $\mathrm{X}$, $\mathrm{Y}, \mathrm{Z}$ coordinates of each of these points to determine size and position with micrometre precision typically.

The research was conducted on extruded aluminium alloys work pieces with $5600 \mathrm{~mm}$ length, used in aerospace industry, with a wide range of alloys and treatments. In Figure 3 is showing the test part placed inside the subassembly. With thin walls and the section represents a high resistance to shock and breakage, develop from advanced alloys tailored for specific applications to help find the right balance of strength, damage tolerance, and corrosion resistance.

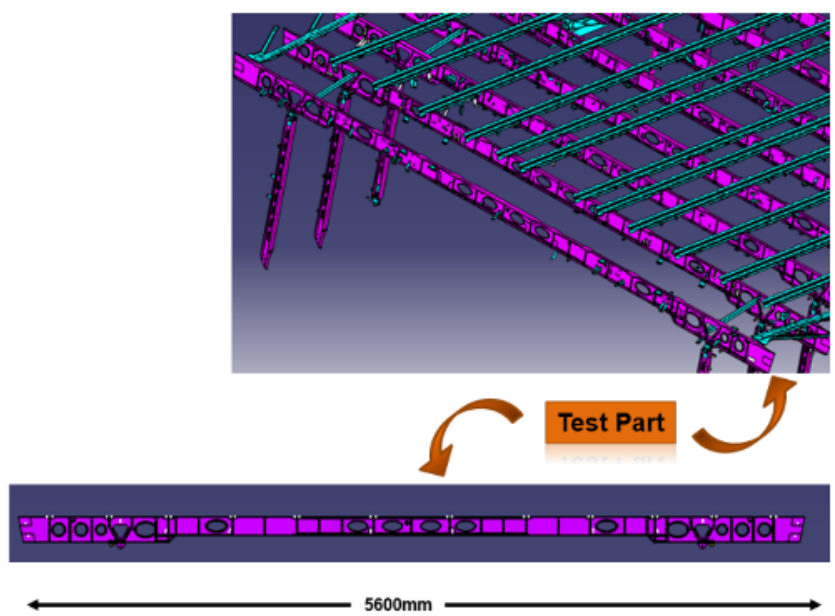

Fig. 3. Aerospace part assembled view.

The problem appears after machining process for those parts on the holes position, which have deviation out of tolerance on X-axis based on the datum reference (Figure 4). The tolerance for the all holes is $\pm 0.2 \mathrm{~mm}$.

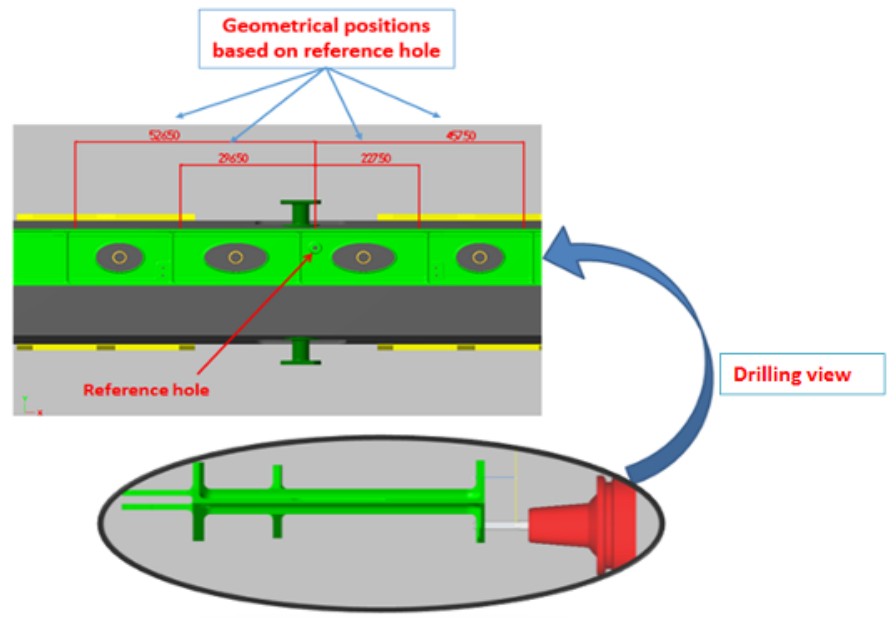

Fig. 4. Machined part view. 


\section{Machining results}

The parts geometry is constrained by one middle hole. After machining four identical pieces, were measured in CMM. In Table 1 are represented the measurement values of the holes geometrical position based on reference hole.

Table 1. First four identical parts machined results.

\begin{tabular}{|c|c|c|c|c|}
\hline $\begin{array}{c}\text { Hole position } \\
\text { on X-axis [mm] }\end{array}$ & P1 & P2 & P3 & P4 \\
\hline-2316.7 & -0.157 & -0.275 & -0.302 & -0.277 \\
\hline-2162.5 & -0.190 & -0.255 & -0.292 & -0.265 \\
\hline-1991.5 & -0.139 & -0.244 & -0.280 & -0.255 \\
\hline-1727.5 & -0.125 & -0.196 & -0.224 & -0.233 \\
\hline-1585.0 & -0.114 & -0.173 & -0.202 & -0.208 \\
\hline-1503.7 & -0.096 & -0.163 & -0.201 & -0.200 \\
\hline-1261.5 & -0.077 & -0.137 & -0.185 & -0.169 \\
\hline-787.5 & -0.058 & -0.081 & -0.111 & -0.092 \\
\hline-457.5 & -0.024 & -0.040 & -0.066 & -0.048 \\
\hline-227.5 & 0.007 & -0.009 & -0.018 & -0.023 \\
\hline 296.5 & -0.093 & -0.066 & -0.056 & -0.067 \\
\hline 526.5 & -0.121 & -0.097 & -0.084 & -0.104 \\
\hline 856.5 & -0.143 & -0.131 & -0.116 & -0.136 \\
\hline 1330.5 & -0.239 & -0.193 & -0.172 & -0.199 \\
\hline 1572.7 & -0.213 & -0.235 & -0.205 & -0.211 \\
\hline 1654.0 & -0.220 & -0.237 & -0.219 & -0.226 \\
\hline 1796.5 & -0.287 & -0.258 & -0.246 & -0.248 \\
\hline 2060.5 & -0.306 & -0.283 & -0.282 & -0.286 \\
\hline 2231.5 & -0.327 & -0.303 & -0.296 & -0.309 \\
\hline 2385.7 & -0.354 & -0.330 & -0.314 & -0.302 \\
\hline
\end{tabular}

By analysing the results it is visible a material contraction to the middle of the part. The maximum deviation value which is on the ends of the part is $-0.354 \mathrm{~mm}$, on chart analysing is visible a polynomial growth (Figure 5).

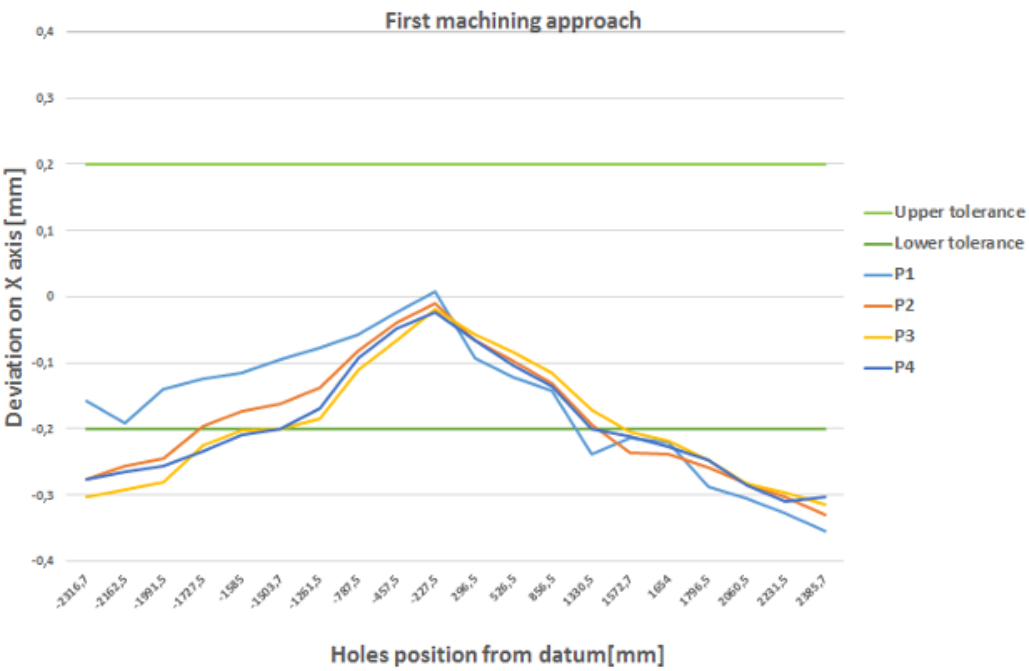

Fig. 5. First four identical parts machined results. 
It is visible the final part contraction at both ends. Through elimination of other factors from Ishikava diagram, which can influence the holes position, was determinate the root cause to by internal stress (Figure 6).

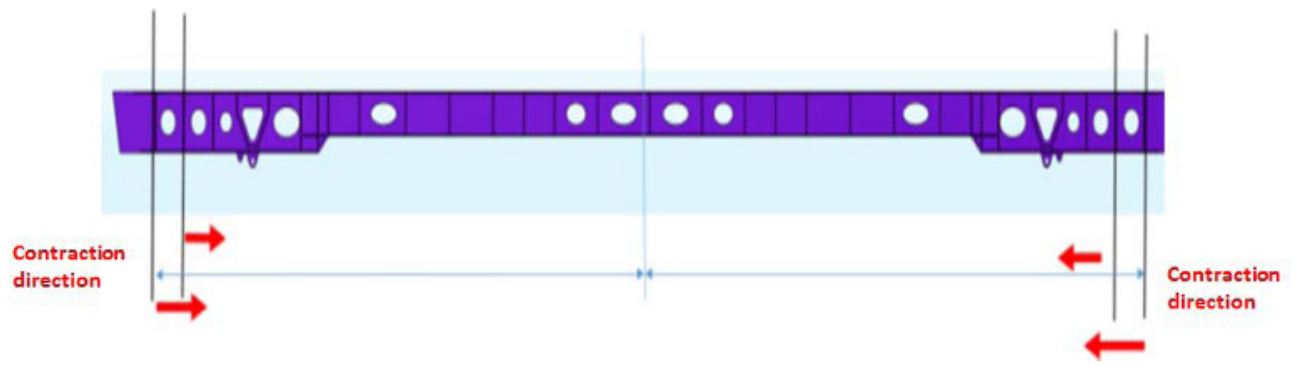

Fig. 6. Part contraction.

The solution for this issue is to eliminate the material stress before drilling operation, that means is to release the part to be on the free state from the clamping device and clamped again after that. Using this approach was made other four identical pieces. In Table 2 are represented the measurement values of the holes position based on the reference hole.

Table 2. Second four identical parts machined results.

\begin{tabular}{|c|c|c|c|c|}
\hline $\begin{array}{c}\text { Hole position } \\
\text { on X-axis [mm] }\end{array}$ & P5 & P6 & P7 & P8 \\
\hline-2316.7 & 0.006 & 0.008 & 0.022 & 0.007 \\
\hline-2162.5 & 0.013 & 0.008 & 0.002 & -0.009 \\
\hline-1991.5 & 0.011 & -0.001 & -0.018 & -0.017 \\
\hline-1727.5 & 0.093 & 0.004 & -0.021 & -0.021 \\
\hline-1585.0 & 0.004 & -0.001 & -0.023 & -0.018 \\
\hline-1503.7 & 0.001 & -0.003 & -0.021 & -0.026 \\
\hline-1261.5 & 0.032 & -0.014 & -0.021 & -0.027 \\
\hline-787.5 & 0.032 & 0.006 & -0.015 & -0.020 \\
\hline-457.5 & 0.032 & 0.035 & 0.004 & 0.009 \\
\hline-227.5 & 0.033 & 0.045 & 0.010 & 0.017 \\
\hline 296.5 & -0.060 & -0.054 & -0.033 & -0.032 \\
\hline 526.5 & -0.078 & -0.057 & -0.040 & -0.037 \\
\hline 856.5 & -0.082 & -0.054 & -0.029 & -0.030 \\
\hline 1330.5 & -0.074 & -0.035 & -0.028 & -0.026 \\
\hline 1572.7 & -0.088 & -0.046 & -0.034 & -0.045 \\
\hline 1654.0 & -0.084 & -0.047 & -0.037 & -0.044 \\
\hline 1796.5 & -0.09 & -0.054 & -0.039 & -0.042 \\
\hline 2060.5 & -0.087 & -0.066 & -0.030 & -0.034 \\
\hline 2231.5 & -0.084 & -0.057 & -0.015 & -0.022 \\
\hline 2385.7 & -0.085 & -0.069 & -0.007 & -0.020 \\
\hline
\end{tabular}

It is visible an improvement on the holes positions. If the internal stress from extrusion is eliminated by releasing the part, the holes are in tolerance (Figure 7). 


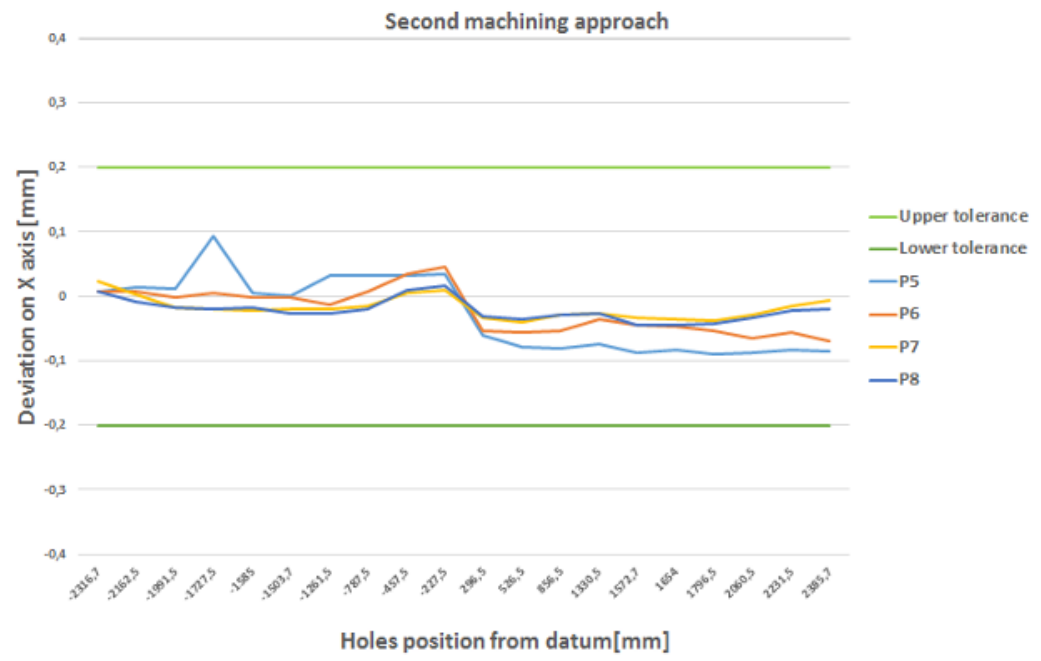

Fig. 7. Second four identical parts machined results.

\section{Conclusions}

This experimental research bring a solution using a simple procedure by releasing the part to be on free state before drilling operation, through this was eliminated the deviations created by internal stress of the extruded profiles. This methodology improves the machining process control and creates a manufacturing repeatability.

Future improvement which creates better precision on the final part will be to apply a temperature coefficient to eliminate the environment temperature fluctuation [5].

The original contribution for this research is: creating a solution to solve the deviations problem.

The experimental research conducted with the support of the machining department of S.C. Universal Alloy Corporation Europe S.R.L., Dumbravita.

\section{References}

1. S..A. Moldovan, Research on Increasing Efficiency in machining on Numerically Controlled Machine at S.C. Universal Alloy Corporation Europe S.R.L., No.3657/09.02.2016 (in Romanian)

2. ***Training Guide PBZ HD Ver. (2015)

3. ***https://www.handtmann.de/en/machining-centres/products/pbz-profile-machiningcentres/pbz-hd/ (2017)

4. ***Metris Bridge CMM User Guide (2016)

5. Ș.A. Moldovan, V. Năsui, Applied Mechanics and Materials 809-810, 33-38 (2015) 